\title{
Development of an alternative method for the calibration of ECG simulators
}

\author{
Roberto Benitez ${ }^{1, \star}$, Romi Uresti ${ }^{2, \star \star}$, and Carolina Solorzano ${ }^{3, \star \star \star}$ \\ ${ }^{1}$ ETALONS, S.A. de C.V., 64640 Monterrey, Mexico
}

\begin{abstract}
In Mexico, the concern on improving the healthcare system has always been of great importance. The relevance of this development is the impact on the improvement of the quality of health system along with human health condition. An ECG simulator oversees calibrating ECG machines, including monitoring, and diagnose. Thus, it is important to be completely sure if an ECG simulator is working according to their specifications, in order to assure the good performance of electrocardiographs. The aim of this method is to describe a calibration procedure for ECG simulators.

A home-designed amplifier was built to amplify the small signal from the ECG simulator for the oscilloscope to display to be able to measure its amplitude and frequency, and compare it with manufacturer's specifications. The amplifier was calibrated using Fluke ProSim 8, as a result a calibration curve and equation was obtained. The restlessness arises from experience and investigations from other authors where they have reported misdiagnosis in certain diseases due to misreading of ECGs, noticing that medical staff base their diagnosis on medical devices. For that reason, an erroneous diagnosis can lead to wrong treatments for patients representing a waste of medical resources and more important potential death.
\end{abstract}

\section{KEYWORDS}

Metrology / Biomedical Engineering / ECG / ECG simulator / Calibration procedure / Amplificator / Misdiagnosis

\section{Introduction}

Etalons is a company that performs calibrations of not only industrial machinery but also medical equipment, always worried about generating new ideas and protocols to calibrate different technologies. Etalons has more than twenty years of experience in metrology field and five years working in the integration of metrology and biomedical sciences,

The importance of this type of procedures is the enhancement of the health system along with the quality of human life as a consequence of the improvement of medical attention.

Next, a calibration procedure for ECG simulators is described along with some cardiophysiology and electronics background needed to completely understand the importance, objective and steps of the calibration of ECG simulators proposed by Etalons.

\subsection{Background}

The heart is a muscle perfectly shaped to work as a pump that creates the necessary force to impulse blood into the

\footnotetext{
${ }^{\star}$ e-mail: roberto@etalons.com.mx

$\star \star$ e-mail: romi.uresti@etalons.com.mx

$\star \star \star$ e-mail: carolina.sb94@hotmail.com
}

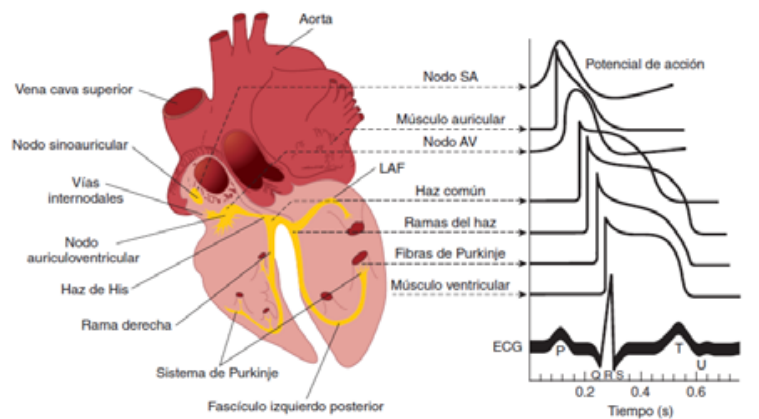

Figure 1: Electrical activity system of the heart. Visual representation of a human heart with its electrical propagation following blood circulation. Each electrical waveform of cardiac cells are represented. The summation of each one of those waveforms according time represents the characteristic ECG wave. Taken from "Origin of the Heartbeat and the Electrical Activity of the Heart", by K. Barret, S.M. Barman, S. Boitano and H.L. Brooks, 2012, Ganong's Review of Medical Physiology, 29, p. 522. Copyright 2012 by the McGraw-Hill Companies, Inc.

whole body. To do that, cardiac muscles have to contract under the command of an electrical stimulus called action potential, which is on the range of 0.2 to $1 \mathrm{mV}$. This action potential spreads around the heart as blood circulates through it. Those changes in action potential during time adds up forming a characteristic wave known as an electrocardiogram [1], (see figure 1).

C) The Authors, published by EDP Sciences. This is an open access article distributed under the terms of the Creative Commons Attribution 
To see and register the electrical activity of the heart, an electrocardiograph (ECG) is used with the help of electrodes placed on the skin at different locations of the body. Each position, commonly known as leads, records differences in potential between two points. There are 12 common leads: precordial leads V1-V6 (see figure 2a), limb leads L1-LIII (see figure 2b) and augmented leads aVR, $\mathrm{aVL}$ and aVF [2]. Each lead has a characteristic wave and amplitude (see figure 3).

Depending on the position of the electrodes, the physician is able to diagnose a disease, predict a heart attack, know the history of the heart and many more heart related information. Any failure can be harmful to the patient or can provoke a misdiagnosis (see section 1.2), this is why it is important to know if the electrocardiogram is working well. To do that, an ECG simulator is needed.

An ECG simulator is a device used to calibrate electrocardiographs by creating the characteristic action potential wave, shown in figure 1 , with electrical components such as resistors and capacitors. Basically, it is a "heart simulator" with small amplitudes and frequencies.

\subsection{Justification}

The importance of these type of experiments and researches is mainly the impact on the improvement of quality of health systems around the world. A non-calibrated device can cause misreadings leading to misdiagnosis and give the patient wrong treatments. Studies, such as (In Bogun F, et al.,) showed that $10 \%$ of total ECG's evaluations were incorrectly mistaken for atrial fibrillation (AFib), affecting $14 \%$ of patients in this experiment [3]. Moreover, 40 patients were affected with a misdiagnosis due to an artifact. It is mentioned that artifacts are the main cause AFib is commonly misdiagnosed. In another article [4], 19\% of ECGs were misdiagnosed with AFib, affecting $35 \%$ of the patients.

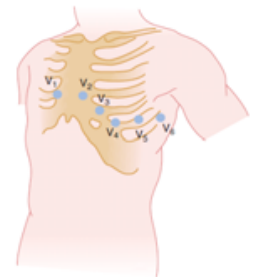

(a) Unipolar leads.

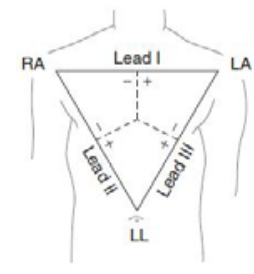

(b) Bipolar leads.
Figure 2: Precordial leads and Einthoven triangle. a) Visual representation of standard unipolar electrode placement on the chest. b) Visual representation of bipolar leads polarization to connect correctly into the body. Taken from "Origin of the Heartbeat and the Electrical Activity of the Heart”, by K. Barret, S.M. Barman, S. Boitano and H.L. Brooks, 2012, Ganong's Review of Medical Physiology, 29, p. 526 and 528. Copyright 2012 by the McGraw-Hill Companies, Inc.

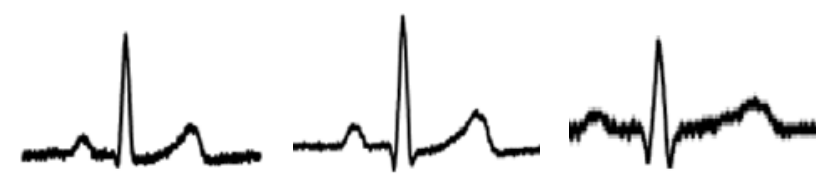

(a) LI

(b) LII

(c) LIII
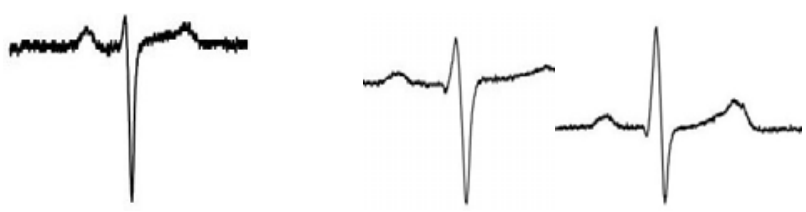

(d) V1

(e) V2

(f) V3

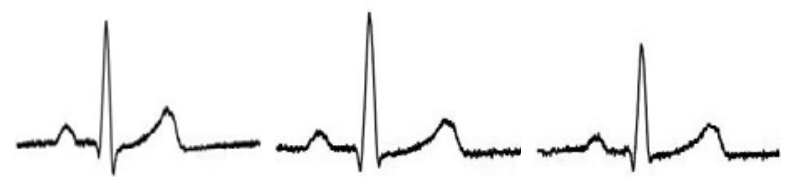

(g) V4

(h) V5

(i) V6

Figure 3: ECG waves representation for each lead. All images for the waveform leads are obtained from the actual amplification of the ECG simulator Fluke ProSim 8 waves, retrieved using the calibration system designed by Etalons. a) LI = Bipolar Lead 1; b) LII = Bipolar Lead 2; c) LIII = Bipolar Lead 3; d) V1 = Unipolar Lead 1; e) V2 = Unipolar Lead 2; f) V3 = Unipolar Lead 3; g) V4 = Unipolar Lead 4; h) V5 = Unipolar Lead 5; i) V6 = Unipolar Lead 6

This is just one particular case involving AFib misdiagnosis, but there are many others where patients were treated for a disease that they don't really have. Thus the importance for calibration of not only a biomedical device but its simulator as well to assure a satisfying functioning.

\subsection{Method Summary}

The following procedure consists of three main components: an ECG Simulator, seen as the unit under test; an amplifier, which is a home-designed circuitry with operational amplifiers (op-amp). This circuitry is used to amplify the small voltages from the ECG simulators for the oscilloscope to display, from $0.2 \mathrm{mV}$ to $1 \mathrm{~V}$ approximately, depending on the brand and model of the ECG simulators different specifications will be found. The third main component is an oscilloscope, a device where characteristic waves are going to be displayed and used to measure amplitudes and periods. Then, data will be collected and analyzed on a special MS-Excel format to know the specific amplification value of the amplifier, get the voltage value from ECG simulators and, finally, obtain a judgment if the unit under test is within the specifications.

On figure $4 \mathrm{a}$ and $4 \mathrm{~b}$, a visual representation and a block diagram of the array to amplify and display the waves from ECG simulators. 


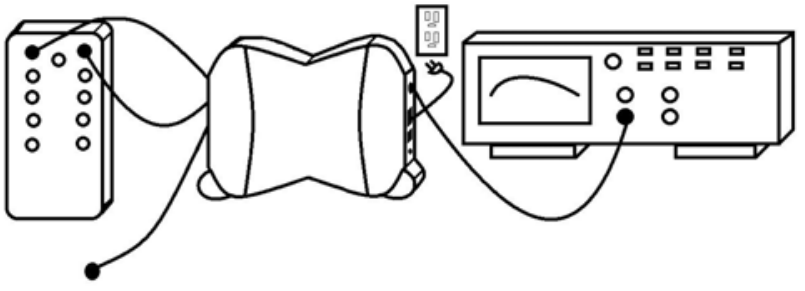

(a) Array for the calibration of ECG simulators.

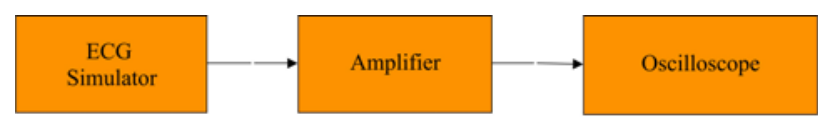

(b) Block diagram for the calibration method of ECG simulators created by Etalons.

Figure 4: Visual representation of the a) array and b) block diagram of the method for ECG simulator calibration

\section{Method}

In order to complete the ECG simulators calibration, a procedure was created. It starts by identifying the type of simulator depending on the amount of leads. As mentioned before, there are 12 different common leads and each one of them has its own characteristic wave and some of them with a positive and negative side which is important to differentiate to place the connections correctly. The most common are 12 leads simulators that have 10 outputs, less common are 3 leads simulators that have 5 outputs.

ECG simulators can give not only the ECG characteristic waveform but also sine, square and triangular waveforms at different frequencies. This will be important to consider for the amplifier's calibration curve.

\subsection{Circuit design for the amplifier}

\subsubsection{Power Supply, amplifiers and filters}

The ECG amplifier consists of an arrange of op-amps to amplify the input signal from the ECG simulator. Each op-amp must be supplied by a power source of around +/$15 \mathrm{~V} \mathrm{DC}$ which is taken from the $120 \mathrm{~V} / 60 \mathrm{~Hz}$ of the power wall, converted to DC using a transformer and corrected to get DC. On figure 5a, the power supply schematic is represented.

Three kinds of op-amps were used to amplify the signal from the ECG simulator. An instrumentation amplifier and two general purpose amplifiers; one with a gain of 1000 and one that operates as a notch filter.

Voltage sources represented as V3 and V4 in figure $5 \mathrm{~b}$ are the ones created with the double power supply discussed previously. Voltage sources represented as V1 and V2 are inputs from the ECG simulator. Vout is the output signal which will be connected to the oscilloscope.

\subsubsection{Amplifier calibration curve}

A calibration curve had to be developed in order to obtain the amplification that the amplifier is giving to ECG

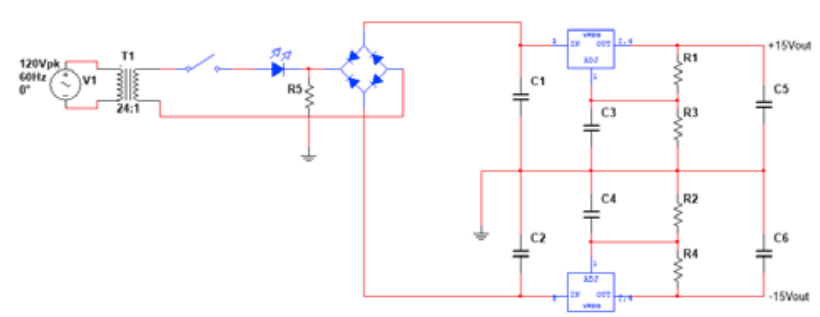

(a) Power supply schematic. Schematic of the double power supply. Done with Multisim.

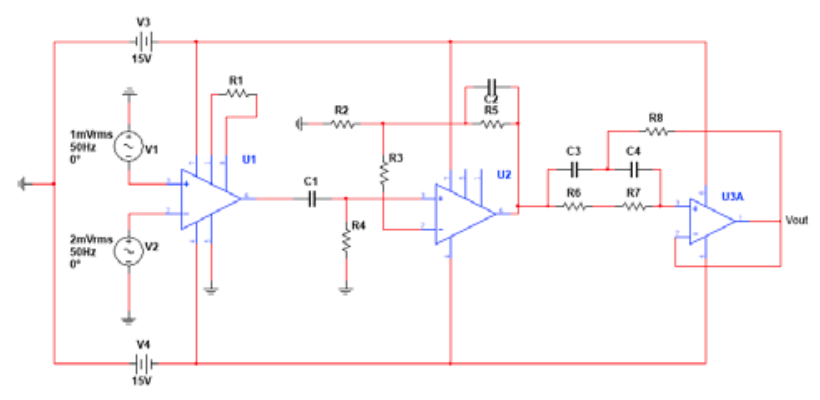

(b) Amplifier schematic. Schematic of operational amplifier with notch filter. Done with Multisim.

Figure 5: Schematics.

simulators and be able to make the comparison between the amplified data and the manufacturer information, better known as nominal data, and finally get the real time amplitudes values.

A graph with amplitude values (see figure 6) was obtained by applying a sine wave of small amplitude within a voltage range between $0.05 \mathrm{mV}$ and $0.5 \mathrm{mV}$ with steps of $0.05 \mathrm{mV}$ and between $0.5 \mathrm{mV}$ and $5 \mathrm{mV}$ with steps of $0.25 \mathrm{mV}$ from a Fluke equipment model ProSim 8 .

The input for the amplifier is represented in the y-axis and the output from the oscilloscope on the x-axis. An Equation 1 was developed showing linear behavior.

$$
y=0.0006 x-0.00008
$$

\subsection{Measurement}

The output signal from the amplifier goes directly to the oscilloscope's input where the measurement is done. Displaying the ECG curve using the oscilloscope gives extra information about the waveform; for example, by observing the exact output waveform, compare it with the physiologically correct to confirm good performance and obtain accuracy for the complete wave not only the highest voltage.

Amplitudes are measured by using the cursor function from the oscilloscope (see figure 7) and connecting the leads following the order presented on table 1

Due to physiological reasons, amplitudes are different on each lead but always following the relation LII > LIII $>$ LI. Depending on the brand and model of the simulator 


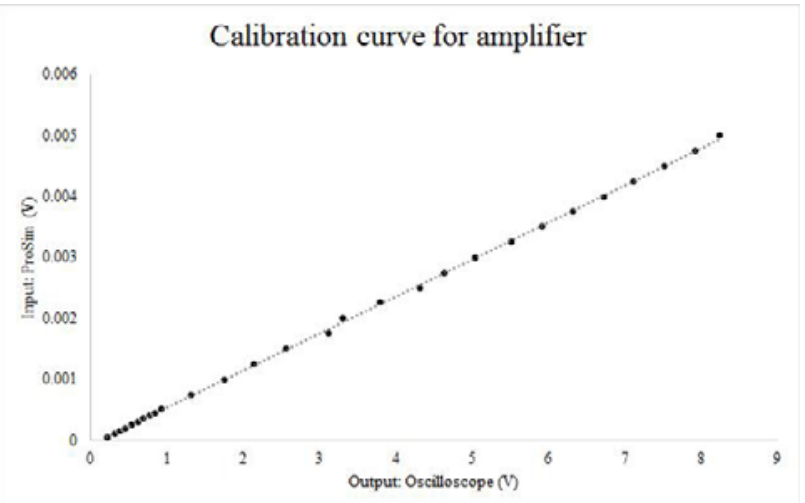

Figure 6: Calibration curve. Calibration curve for the home-designed amplifier. It was done by taking as input the sinusoidal waves from Fluke ProSim 8 within the voltages amplitudes of an ECG simulator, displaying the output on the oscilloscope to measure amplitudes. By knowing the input to the amplifier, calculations could be done to obtain the exact amplification.

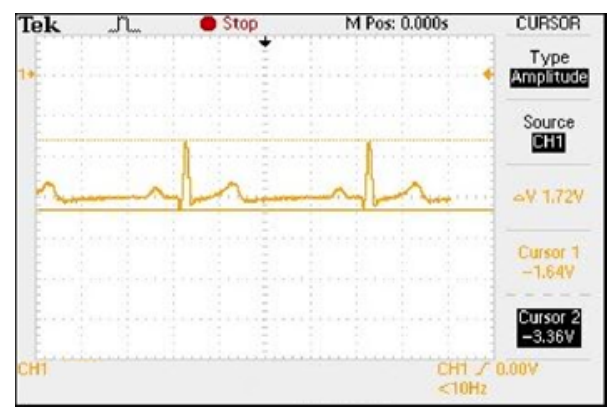

Figure 7: Amplitude cursor function on oscilloscope.

Table 1: Lead connection according to its polarity following the Einthoven Triangle. Note: Ref = Reference lead; RL = Right Leg; RA = Right Arm; LA = Left Arm; LL = Left Leg; LI = Lead number 1; LII = Lead number 2; LIII = Lead number 3; V1 = Unipolar lead number 1; V2 = Unipolar lead number 2; V3 = Unipolar lead number 3; V4 = Unipolar lead number 4; V5 = Unipolar lead number 5; V6 = Unipolar lead number 6.

\begin{tabular}{c|c|c|c|c|c} 
Ref:RL & + & - & & + & - \\
\hline LI & RA & LA & V3 & LA & V3 \\
LII & LL & RA & V4 & LA & V4 \\
LIII & LA & LL & V5 & LA & V5 \\
V1 & LA & V1 & V6 & LA & V6 \\
V2 & LA & V2 & & & \\
\hline
\end{tabular}

V1 to V6 can have different behaviors, for example, they can change from smaller to greater or smaller to greater and again smaller.

When doing measurements on the oscilloscope, amplitude measurement is done in Volts on the y-axis on each one of the leads. For frequency measurement, it is done in Hertz on the x-axis, specifically, in 60 BPM in the LII, because frequencies in all leads are the same. To be sure that the given frequency matches with the one established by the manufacturer specifications in BPM (Beats Per Minute), some conversions have to be done.

Where $T=\frac{1}{f}$, then $B P M=T * 60$. On the next table (see table 2), a summary of the oscilloscope measurement is shown for better understanding of what is being measured.

Five repetitions of the measurements from LI to V6 are done to complete the calibration, an average is obtained. Then, by using equation 1 , the specific amplification for each of the leads are calculated. This represents the corrected voltage that is going to be compared with the nominal value. Also, on the same format uncertainty is calculated.

Even for equipments that do not have manufacturer specifications, either for obsolescence or lack of information, these calculation can still be performed.

\subsection{Uncertainty Calculation}

Given the definition of uncertainty from the International Vocabulary of Metrology by the JCGM, where uncertainty is a parameter that cannot be negative and characterize the dispersion of certain values associated to a standard or measurand, including corrections and values of standards measurement uncertainty already given by a certificate of calibration [5]. To complete the calculation of uncertainty of a given measured value, there has to be included the uncertainty added by all the equipment that helps to get information from a certain unit under test or instrument that is going to be calibrated, in this case ECG simulators.

The uncertainty added to the voltage and frequency measurement of ECG simulators comes from, in order of appearance, the amplifier which includes uncertainty from the small sinusoidal voltage generator Fluke ProSim 8 and the oscilloscope where the ECG wave is displayed; also, the expanded uncertainty includes the uncertainty added by the equation calculation, the amplification factor uncertainty and the oscilloscope's uncertainty. Figure 8 shows a diagram to visualize the uncertainty calculations for better understanding.

Equations 2 and 3 are to calculate the combined uncertainty of the amplifier, given the data from the calibration of the amplifier with the sine wave input from Fluke ProSim 8 and output observed in the oscilloscope after the amplifier. Both equations include the uncertainty given by the repeatability of the measurement, the resolution

Table 2: Oscilloscope measurement, axes and units. Note: $\mathrm{Hz}=$ Hertz; $\mathrm{BPM}=$ Beats Per Minute.

Oscilloscope measurement

\begin{tabular}{c|c|c}
\hline Amplitude & y-axis & Volts \\
\hline Frequency & x-axis & $\mathrm{Hz}->\mathrm{BPM}$ \\
\hline
\end{tabular}



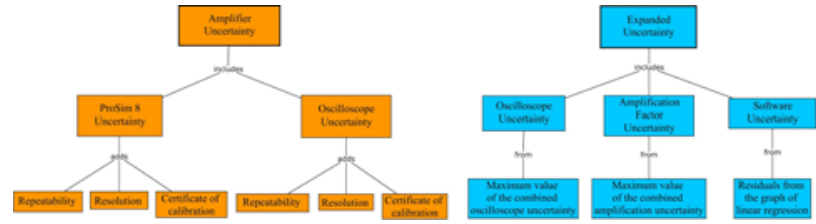

Figure 8: Diagram for ECG simulators uncertainty calculations.

of the standard that is taking the measures and the value for uncertainty given at the certificate of calibration from the manufacturer specifications. Equation 4 represents the combined uncertainty adding the coefficient of sensitivity for each of the uncertainty values from ProSim 8 and oscilloscope.

$$
\begin{gathered}
\mu_{\text {ProSim }}=\sqrt{\mu_{r e p}^{2}+\mu_{c e r t}^{2}+\mu_{r e s}^{2}} \\
\mu_{O s c}=\sqrt{\mu_{r e p}^{2}+\mu_{c e r t}^{2}+\mu_{r e s}^{2}} \\
\mu_{\text {Amp }}=\sqrt{\left(\mu_{\text {ProSim }} * C_{i \text { ProSim }}\right)^{2}+\left(\mu_{O s c} * C_{i O s c}\right)^{2}}
\end{gathered}
$$

To obtain the expanded uncertainty more equations have to be found. Equation 5 is to obtain uncertainty of the amplification factor (AmpF) by taking the maximum value from the combined amplification uncertainty obtained before. Equation 6 is for uncertainty of the oscilloscope to finally get the expanded uncertainty, it is obtained by taking the maximum value of the combined oscilloscope uncertainty that was calculated before. To obtain the Equation 1 and the calibration curve, MS-Excel was needed for data processing because this procedure adds uncertainty Equation 7 [6] had to be established, which is determined by evaluating the standard deviation of residuals errors, for the whole interval of data [6].

$$
\begin{aligned}
& \mu_{A m p F}=\max \left(\mu_{A m p}\right) \\
& \mu_{\text {Osc } 1}=\max \left(\mu_{\text {Osc }}\right) \\
& \mu_{\text {Residuals }}=\sqrt{\frac{\sum_{i=1}^{N}\left(\text { Residual }_{i}\right)^{2}}{N-m-1}}
\end{aligned}
$$

To obtain the combined uncertainty for each lead of ECG simulators, Equation 8 was developed along with the coefficients of sensitivity for the uncertainty of the amplifier factor, the oscilloscope and the uncertainty from the standard deviation of residuals errors. The final Equation 9 is to obtain the expanded uncertainty which is done by multiplying by 2 the uncertainty from the ECG simulators in order to include the $95 \%$ of data given certain standard deviation, using a coverage factor $(\mathrm{k})$ equal to two.

$$
\begin{gathered}
\mu_{E C G s i m}=\sqrt{\left(\mu_{A F} * C_{i A F}\right)^{2}+\left(\mu_{O s c 1} * C_{i O s c 1}\right)^{2}+\mu_{\text {Residuals }}^{2}} \\
U_{\text {ECGsim }}=\mu_{\text {ECGsim }} * 2, \text { for }: k=2
\end{gathered}
$$

\section{Results}

\subsection{Advantages}

The main advantage of this project is the low-cost building. For that reason, the consideration of this project as an excellent prototype to design and build for academic undergraduate students such as biomedical or electrical engineers in order to understand some principles of metrology, and at the same time apply and combine electrical and human physiology concepts.

The system can be easily transport to calibrate off-site equipment when needed. Once you completely understand the concept, the importance and the physiological aspects; it is really easy to replicate and systematize the process of calibration.

\subsection{Problems encountered and improvements}

During the experimentations and development of the method some problems were encountered and areas of opportunity were identified for further work.

One of the main problems that were found when performing the first tests of the amplifier prototype was a phase change in the output signal on the oscilloscope. It is not a problem that affects the measurements, since the oscilloscope has a function to change the signal position to better view. However, this problem can be improved in the future.

All of the circuits were done on copper breadboards and hand-welded. Due to this, the amplifier became a fragile object, although its case protects it very well and isolates external noise. Other mechanical waves or even loud sounds can have an affection on the signal output, but most of the time the amplifier prototype performs adequately. This can be fixed by printing the circuits and weld the components with a special machine to avoid mistakes to reduce any external noise and fragility.

The most important problem that was overcome When developing the calibration curve of the amplifier was; firstly, that the amplifier calibration was tried to be done with a wave generator that was not able to give voltages lower than $1 \mathrm{mV}$, due to that all data under $1 \mathrm{mV}$ were excluded and miscalculations inside that range were found. To solve that problem, a sine wave source able to give voltages under $1 \mathrm{mV}$ was located in the Fluke ProSim 8 system and applied to the amplifier to obtain good information inside the ECG simulator voltage range.

All ECG simulators are different and give different ranges of voltages amplitudes, for that reason it cannot be possible to assume data and it is completely necessary to look for manufacturer manuals in order to get those values. Commonly, those values could be in reference to LI or LII, given in percentages. If the ECG simulator is old or obsolete to find its manufacturer manual becomes an obstacle.

\section{Summary}

This paper discussed physiological and electronics principles of an ECG and ECG simulators to fully explain and 
understand the calibration procedure for an ECG simulator. This method required the building of an electrical amplifier to see the waveform on an oscilloscope in real time and measure ECG parameters, such as amplitude and period. It also required extensive uncertainty calculations in order to have a proper calibration. The whole system (p.e. amplifier) can be built by electrical or biomedical students with basic knowledge on cardiovascular physiology and electronics, meaning this could be optimal for learning purposes on biometrology.

Biometrology is an unexplored area of study which has a lot of potential for improvement the medical attention at hospitals and clinics. During all the process, since the entry to the hospital, the patient, is in constant interaction with medical equipment that must be calibrated to assure its good performance and avoid any misreading and misdiagnoses.

\section{References}

[1] J. Carr, J. Brown, eds., Electrocardiography, Vol. 4 of Introduction to Biomedical Equipment Technology. (Prentice Hall, New Jersey, EUA, 2001)

[2] K. Barret, S. Barman, S. Boitano, H. Brooks., eds., Origin of the heartbeat and the electrical activity of the heart., Vol. 24 of Ganong's Review of Medical Physiology. (McGraw Hill., New York, EUA, 2012)

[3] F.M. Bogun, R. Bazzi, E. Wissner, G. Kalahasty, C. Schuger, eds., Atrial Fibrillation: Misdiagnosis and Clinical Consequences (J Am Coll Cardiol, 2003)

[4] F.M. Bogun, D. Anh, G. Kalahasty, E. Wissner, C. Serhal, R. Bazzi, C. Schuger, eds., Misdiagnosis of Atrial Fibrillation and Its Clinical Consequences. (The American Journal of Medicine, 2004)

[5] JCGM, ed., "Uncertainty”. (International Vocabulary of Metrology., Geneva, Switzerland, 2012)

[6] MetAs., ed., Linealidad; Curvas de ajuste, Interpolación y Extrapolación. (La Guía MetAs., D.F., Mexico, 2008) 